\section{MS}

médecine/sciences $1991 ; 7: 208-10$

\title{
LE DIABĖTE VAINCU AVANT L'AN 2000?
}

\section{Pierre Lefèbvre}

\section{ADRESSE}

P. Lefèbvre : professeur à l'Université de Liège, Président de l'European Association for the Study of Diabetes. Département de Médecine, Service de Diabétologie, Nutrition et Maladies Métaboliques, Domaine Universitaire du

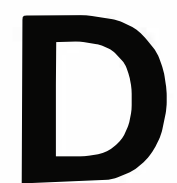

ès la fin du XIX ${ }^{e}$ siècle, se dessine dans le domaine du diabète sucré une évolution des connaissances dont l'accélération au cours des dernières années du XXe siècle peut être qualifiée de prodigieuse. Entre 1845 et 1870, Claude Bernard établit les bases de la régulation du métabolisme glucidique ; en 1869, Langerhans défend à Berlin une thèse où il décrit, au sein du pancréas, de petits amas cellulaires se différenciant du parenchyme exocrine ; en 1889, à Strasbourg, von Mehring et Minkowski reproduisent expérimentalement le diabète par pancréatectomie chirurgicale chez le chien. A l'aube de ce siècle, naît l'endocrinologie et de Meyer, à Bruxelles, baptise du nom d'insuline une hormone encore hypothétique mais dont il préssent l'origine dans certaines des cellules des îlots décrits par Langerhans. Paulesco, à Bucarest, et Banting et Best, à Toronto, co-découvrent l'insuline en 1921. Le premier patient, Leonard Thompson, est traité par insuline le 11 janvier 1922. Une ère nouvelle est ouverte, une maladie jusque-là mortelle peut être soignée avec succès. Grâce à l'insuline, certains patients ont survécu plus de 60 ans à la découverte de leur diabète !

Les insulines sont successivements purifiées, modifiées ou combinées à divers agents pour en prolonger la durée d'action, purifiées encore pour être débarrassées de divers contaminants. Sanger obtient le Prix Nobel pour la description de la structure primaire de la molécule d'insuline. Berson et Yalow décrivent en 1959-1960 le premier dosage radio-immunologique d'une substance biologique, l'insuline toujours. Cette découverte, technique, ouvre un champ prodigieux d'investigation en biologie et confirme ce que le clinicien savait depuis longtemps : il y a deux grandes formes de diabète idiopathique. L'une, diabète de type 1 (ou insulinodépendant) est caractérisée par une carence insulinique, laquelle est mortelle lorsqu'elle est totale. L'autre, diabète de type 2 (ou non insulinodépendant) se caractérise par la persistance de taux normaux, voire, dans certaines circonstances, accrus, d'insuline dans le sang: de toute évidence il y a insulinorésistance!

Berston étant décédé en 1972, c'est Rosalyn Yalow seule qui est couronnée en 1977 par un Prix Nobel de médecine. Steiner élucide les mécanismes de la biosynthèse de l'insuline en découvrant son précurseur obligé, la pro-insuline. L'industrie pharmaceutique réalise la production industrielle d'insuline humaine par génie génétique en programmant le génome d'E. Coli ou de Saccharomyces cerevisiae. Cette énumération, rapide et incomplète, illustre notre propos liminaire : le diabète a été directement ou indirectement à la base d'une série impressionnante de découvertes fondamentales en biologie et en médecine. Et pourtant, 70 ans après la découverte de l'insuline, le bilan reste terrifiant. Le patient atteint de diabète de type 1 est, sensu stricto, insulinodépendant. Deux heures après l'interruption d'une perfusion sous-cutanée continue d'insuline, la dégradation métabolique est évidente, six heures après hyperglycémie et cétose sont majeures; le coma intervient en 24-48 heures et la mort est inéluctable si l'on ne reprend l'administration d'insuline : miracle de l'insulinothérapie, précarité de la santé du patient privé quelques heures seulement de l'hormone à laquelle il doit la vie. Même traité, le patient diabétique a souvent une espérance de vie réduite, il a dix fois plus de risque 
de devenir aveugle et deux à trois fois plus de risque de faire un infarctus du myocarde qu'un sujet non diabétique de même âge. Le problème social est considérable. En France comme en Belgique, la prévalence du diabète de type 1 est de l'ordre de 1 pour 400 avec une incidence annuelle (nombre de nouveaux cas par an) atteignant 7 à 8 pour 100000 . On dénombre $2 \%$ de la population présentant un diabète de type 2 connu et toutes les enquêtes indiquent que pour chaque patient atteint de diabète de type 2 connu et diagnostiqué, il y en a probablement un dont la maladie, totalement silencieuse, est ignorée! Les contributions rassemblées dans ce numéro par la Rédaction de médecine/sciences nous indiquent que les espoirs sont grands d'aboutir, avant la fín du siècle, à un bouleversement de nos connaissances de la pathogénie des diabètes et, potentiellement, à des traitements non plus simplement substitutifs mais cette fois préventifs voire curatifs. Le titre de la contribution de C. Boitard et J.F. Bach (p. 226) est éloquent dans sa concision et sa précision : le diabète insulinodépendant (diabète de type 1) est une maladie polygénique d'origine auto-immune. Ils l'illustrent en résumant les observations effectuées chez l'homme et plus encore par l'étude de deux modèles remarquables de diabète insulinodépendant spontané observé chez l'animal, que ce soit la souris NOD ou le rat BB. On sait depuis une dizaine d'années que, dans nos populations européennes dites " caucasiennes ", 90 à $95 \%$ des patients atteints de diabète de type 1 sont HLA DR3 ou DR4 ou encore hétérozygotes DR3/DR4; dans ce dernier cas, le risque relatif de développer un diabète de type 1 est de l'ordre de 40, c'est-à-dire que des individus porteurs à la fois des phénotypes DR3 et DR 4 ont 40 fois plus de risque de développer un diabète de type 1 que ceux qui ne sont ni DR3 ni DR4. La prévalence dans nos populations du diabète de type 1 étant, nous l'avons vu, de l'ordre de $0,25 \%$, un sujet DR3/DR4 a $0,25 \times 40$ soit $10 \%$ de risque de développer un jour un diabète de type 1 . A vrai dire, comme cela est développé dans la contribution de $\mathrm{m} / \mathrm{s} n^{\circ} 3$, vol. 7, mars 91
I. Khalil et al. (p. 235), les spécificités en cause ont pu être mieux définies grâce à l'avènement des techniques de biologie moléculaire. On sait maintenant que certains allèles $\mathrm{DQ}$ dont les loci sont voisins des allèles $\mathrm{DR}$, et en fort déséquilibre de liaison avec eux, sont en fait en cause. Ces auteurs proposent un modèle moléculaire séduisant dans lequel un rôle essentiel serait joué par deux régions diamétralement opposées aux deux extrémités de la cavité engendrée par les chaînes $\alpha$ et $\beta$ de la molécule HLA DQ. La susceptibilité à la maladie serait régie par une gamme de degrés de risque selon la densité des séquences moléculaires conférant un risque accru (Arg 52 $\mathrm{DQ} \alpha$ ) ou une protection (Asp $57 \mathrm{DQ}$ $\beta)$. Selon ces auteurs, ce modèle " suggère un mécanisme moléculaire d'affinité pour un peptide encore hypothétique qui serait anormalement présenté aux mécanismes inducteurs de la réponse immune ". La nature biochimique de l'antigène cible de la réaction auto-immune contre les îlots de Langerhans est inconnue comme le soulignent et le discutent C. Boitard et J.F. Bach.

Quoiqu'il en soit, et comme l'analysent J.C. Carel et al. (p. 240), il est possible aujourd'hui de dépister de façon précoce le diabète de type 1 longtemps avant son expression ultime, l'élévation de la glycémie. Semblable dépistage comporte un volet génétique déjà mentionné, et un volet immunologique, à savoir la mise en évidence des marqueurs d'un phénomène d'auto-immunité dirigée contre les îlots de Langerhans du pancréas (anticorps anti-îlots de Langerhans, auto-anticorps dirigés contre l'insuline, anticorps dirigés contre un antigène protéique de $64 \mathrm{kDa}$ des îlots humains, etc.). Un premier dommage apporté à la masse des cellules $\beta$ des îlots de Langerhans peut être décelé par des tests métaboliques : réponse insulinique déficitaire à une surcharge glucosée intraveineuse ou mise en évidence d'anomalies de la pulsatilité physiologique de la sécrétion de l'insuline. L'intérêt d'un tel dépistage ultraprécoce du diabète de type 1 est plus qu'académique : il a déjà permis la mise en place d'études contrôlées tentant d'enrayer la destruction progressive des cellules $\beta$ des îlots de Langerhans et, par là, de retarder l'apparition de la maladie dans son expression clinique. A terme, l'objectif est plus ambitieux encore : on peut imaginer, comme l'écrivent J.C. Carel et al. que "la connaissance des autoantigènes impliqués dans le processus auto-immun anti-cellules $\beta$ et l'émergence d'une nouvelle génération d'immunosuppresseurs permettront l'induction d'une tolérance spécifique vis-à-vis de ces antigènes, une véritable vaccination contre la maladie ". Le diabète de type 1 était mortel jusqu'en 1921, on peut aujourd'hui le soigner mais non le guérir, si l'on fait exception des tentatives de transplantations de pancréas et d'îlots de Langerhans. Il est permis de penser que l'on pourra, dans les trois à quatre années qui viennent, intervenir à un stade préclinique et retarder voire prévenir l'éclosion de la maladie. L'objectif ultime, la prévention du diabète par induction d'une tolérance spécifique vis-à-vis du ou des autoantigène(s) impliqués dans la destruction des îlots de Langerhans, n'est pas encore en vue mais l'intense activité de recherche dont témoignent les contributions de ce numéro de médecine/sciences indique un avenir prometteur à moyenne échéance.

L'enthousiasme engendré par les résultats exceptionnels de la recherche sur l'étiopathogénie du diabète de type 1 ne doit pas nous faire perdre de vue que, dans nos pays, 80 à $90 \%$ des patients sont diabétiques tout en gardant une sécrétion insulinique très significative. Ces patients sont atteints de diabète de type 2 ou diabète non-insulinodépendant (DNID). Chanson et al. ( $\mathrm{m} / \mathrm{s} n^{\circ} 4$, vol. 7, avril 91) nous en feront pénétrer les mystères. Ce diabète est fréquent, il touche dans nos pays 3 à $5 \%$ de l'ensemble de la population, il est souvent méconnu : de nombreuses études de dépistage indiquent, comme nous l'avons déjà signalé, que pour un cas de DNID connu, il existe en moyenne un cas où le diabète est totalement ignoré, en raison du caractère insidieux de la symptomatologie. La maladie a une importante composante génétique (la concordance est la règle chez les 
jumeaux univitellins) mais à ce jour la nature $\mathrm{du}$, ou plus probablement des gènes en cause, n'a pu être établie. Cette forme de diabète associe un certain degré d'insulinorésistance et un défaut de l'insulinosécrétion. La controverse reste entière en ce qui concerne l'anomalie initiale. En fait, insulinorésistance et déficit de sécrétion insulinique sont tous deux générateurs d'hyperglycémie, laquelle aggrave l'insulinorésistance et réduit les capacités sécrétoires des cellules $\beta$ du pancréas: ainsi se construit un cercle vicieux où l'hyperglycémie chronique aggrave la situation métabolique.

L'état d'insulinorésistance du diabète de type 2 se caractérise par un défaut de captation périphérique et de stockage de glucose (essentiellement sous la forme de glycogène dans les muscles) et par un débit glucosé hépatique accru. L'anomalie moléculaire responsable de ces altérations reste totalement inconnue. L'hypothèse d'une anomalie siègeant au niveau de l'activité tyrosine-kinase de la sousunité $\beta$ du récepteur insulinique est souvent avancée mais certains travaux récents suggèrent qu'ici encore l'anomalie, significativement corrigée par un traitement insulinique, pourrait être secondaire à l'hyperglycémie chronique.

I.'hypothèse d'une anomalie initiale fonctionnelle au niveau des cellules $\beta$ des îlots de Langerhans ne peut être exclue. La complexité des mécanismes en cause dans le contrôle de l'insulinosécrétion est bien mise en évidence dans la contribution de $\mathrm{B}$. Portha (p. 212). Le lecteur aura compris qu'il est, à ce jour, beaucoup plus difficile d'aborder rationnellement le traitement du diabète non insulinodépendant. Parfois la correction d'un facteur bien précis d'insulinorésistance, un excès pondéral par exemple, permet d'améliorer la situation. Souvent la mesure ne suffit pas, le clinicien tente alors de stimuler la sécrétion endogène d'insuline par les sulfonylurées, d'améliorer l'utilisation du glucose par les biguanides, de réduire les excursions glycémiques post-prandiales par le recours à des inhibiteurs des hydrolases digestives... souvent il est acculé à prescrire de l'insuline à un patient qui en sécrète encore (et peut être, ce faisant, d'accroître le risque d'athérosclérose), il estime alors que son patient est devenu "insulinorequérant " ou "insulinonécessitant". Souvent hélas, toutes ces mesures ne suffisent pas à contrôler le diabète et à prévenir les complications secondaires graves de la maladie.

En conclusion, au seuil de la dernière décennie de ce siècle, patients et médecins peuvent entrevoir la guérison, voire la prévention du diabète de type 1 (diabète insulinodépendant). La transplantation des îlots de Langerhans est la voie la plus prometteuse du traitement substitutif de la maladie établie (voir l'article de D. Pipeleers, $\mathrm{m} / \mathrm{s} n^{\circ} 4$, vol. 7, avril 1991). La connaissance du terrain génétique et la détection précoce d'une attaque auto-immune des îlots de Langerhans devraient permettre des interventions thérapeutiques visant à enrayer le cours de l'affection, et ce à un stade pré-clinique. A terme, la connaissance des autoantigènes impliqués dans la destruction des cellules $\beta$ des îlots de Langerhans devrait permettre d'envisager l'induction d'une tolérance spécifique vis-à-vis de ces molécules. En revanche, en ce qui concerne le diabète de type 2 ou diabète non-insulinodépendant, le chemin à parcourir est probablement plus long. Il s'agira de définir s'il s'agit d'une maladie univoque ou, comme le pensent beaucoup, d'un syndrome hétérogène où la maladie peut débuter aussi bien par une anomalie de la sensibilité à l'insuline que par un déficit de la sécrétion de l'hormone. Dans l'un comme dans l'autre cas, insulinorésistance et troubles de l'insulinosécrétion peuvent être imbriqués, car aggravés par l'hyperglycémie induite. Comme dans le cas du diabète de type 1 , une meilleure connaissance des anomalies moléculaires à la base du (ou des) diabète(s) de type 2 est un préalable indispensable à une thérapeutique rationnelle de cette maladie si largement répandue

\section{TIRÉS A PART}

P. Lefèbvre. 